\title{
Winning, Losing and Drawing in Concurrent Games with Perfect or Imperfect Information
}

\author{
Glynn Winskel \\ Computer Laboratory, University of Cambridge, UK
}

\begin{abstract}
Nondeterministic concurrent strategies - those strategies compatible with copy-cat behaving as identity w.r.t. composition - have been characterised as certain maps of event structures. This leads to a bicategory of general concurrent games in which the maps are nondeterministic concurrent strategies. This paper explores the consequences of extending concurrent games with (1) winning, losing and, implicitly, neutral configurations, and (2) access levels, to address situations where Player or Opponent have imperfect information as to what has occurred in the game. In both cases winning strategies are shown to form bicategories of games. The bicategories become equivalent to order-enriched categories when restricted to deterministic strategies.
\end{abstract}

\section{Introduction}

Dexter Kozen is an inspiring speaker, enjoys teaching, and has a history of involving students, including undergraduates, in research. We share a close association with the Computer Science department at Aarhus, Denmark, going back over several decades - we first met there in 1979. This paper, in Dexter's honour, is based on a recent student project from a lecture course on concurrent games I gave in Aarhus last summer (August-September, 2011) [1].

Its roots lie in John Conway's "Numbers and Games" [2]. There Conway defined his "surreal numbers" as strengths of certain games: he defined a preorder between games $G$ and $H$ if a winning strategy for $G$ gives rise to a winning strategy for $H$; the surreal numbers appeared as equivalence classes induced by the preorder. Shortly afterwards André Joyal uncovered a category underpinning Conway's work. Conway's games support two important operations on two-party games: a form of parallel composition $G \| H$, which Conway called a sum of games; a dualizing operation $G^{\perp}$ which reverses the roles of Player and Opponent in $G$, which Conway called negation. Joyal, following the method used in Conway's proofs, defined a strategy $\sigma$ from a game $G$ to a game $H$, written $\sigma: G \rightarrow H$, to be a strategy $\sigma$ in $G^{\perp} \| H$. Joyal showed that strategies compose, with identities given by copy-cat strategies. A strategy in $H$ corresponds to a strategy from the empty game $\varnothing$ to $H$. Note that

$$
\varnothing \rightarrow \mapsto \rightarrow \mapsto H \text { composes to give } \varnothing \rightarrow \mapsto H,
$$


so a strategy in $G$ gives rise to a strategy in $H$ when there is a strategy from $G$ to $H$. Conway's pre-order between games $G$ and $H$ is witnessed through the presence of a winning strategy of from $G$ to $H$.

This article takes the ideas of Conway and Joyal into the realm of concurrent/distributed processes. It brings the experience of concurrency (event structures, stable families, their techniques and constructions originally used in the semantics of process languages [3]) to bear on the theory of games. It considers a very general definition of 2-party concurrent games in which Player (more accurately thought of as a team of players) competes against Opponent (a team of opponents) in a potentially highly-distributed fashion, without for instance insisting on the alternation of Player and Opponent moves. For most of the article the games will be games of perfect information, in that Player can see all moves of Opponent, and vice versa. An example of such a concurrent game would be simultaneous chess, possibly with collaboration between players. However, the dichotomy Player/Opponent can also be read as process/environment, proof/refutation, or ally/enemy, and there are many other examples of concurrent games in Computer Science and Logic, and beyond.

The methodology is essentially that of Joyal, following Conway, developed within a general model for concurrent computation. Two-party games and strategies are represented as event structures with polarity, in which polarities distinguish the moves of Player and Opponent- $c f$. [4]. A pre-strategy is a total map $\sigma: S \rightarrow A$ of event structures with polarity. The map expresses how moves of Player and Opponent, the events of $S$, correspond to the moves permitted by the game, the events of $A$; that $\sigma$ is a map ensures that play of respects the constraints of the game. Following Joyal, a pre-strategy from a game $A$ to a game $B$ is understood as a pre-strategy in a composite game got by setting the dual game of $A$, reversing the roles of Player and Opponent, in parallel with $B$. From this general scheme nondeterministic concurrent strategies - pre-strategies for which copy-cat strategies behave as identities w.r.t. composition of pre-strategies - have recently been characterized as those pre-strategies which satisfy the two conditions of receptivity and innocence [5]. The extension with winning conditions and the question of when and whether concurrent games are determined (i.e. there is either a winning strategy for Player or Opponent) is considered in the Aarhus lecture notes [1] and the forthcoming article [6]. The two contributions of this paper are: (1) an extension of the framework to games with neutral positions, which as outcomes of a play yield a draw (the student project at Aarhus); (2) an extension to concurrent games with imperfect information, where moves may be hidden, so cannot be taken account of by strategies. Where the article builds on earlier results proofs can be found in the Aarhus lecture notes [1].

A word on related work. A general motivation has been the search for a form of generalized domain theory suitable for the semantics of concurrent processes and proofs [7]. An early definition of concurrent games appears in [8], where Samson Abramsky and Paul-André Melliès presented deterministic concurrent strategies as, essentially, partial closure operators on the domain of configurations of an event structure; such an operator takes any reachable configuration 
of the game to the result of playing the intended moves of Player. Their motivation was the representation of proofs in linear logic. There followed a battery of insightful papers by Melliès and colleagues on asynchronous games culminating in the definition of ingenuous strategies - see e.g. [4,9]. The receptive ingenuous strategies of Melliès and Samuel Mimram have been shown to coincide with the deterministic concurrent strategies of [5], so justifying receptive ingenuous strategies as the most general deterministic concurrent strategies for which copycat behaves as identity. In comparison with early work of Abramsky and Martin Hyland on winning conditions in sequential games, the work here is closer to Hyland's, which it can be seen as extending $[10,11]$. The extension to games of imperfect information was guided solely by the wish to handle such games in a way that respected the bicategorical structure on concurrent games. There are however striking similarities with work by Abramsky and Radha Jagadeesan on games for access control [12].

\section{Event structures and stable families}

An event structure comprises ( $E$, Con, $\leq)$, consisting of a set $E$, of events which are partially ordered by $\leq$, the causal dependency relation, and a nonempty consistency relation Con consisting of finite subsets of $E$, which satisfy

$$
\begin{aligned}
& \left\{e^{\prime} \mid e^{\prime} \leq e\right\} \text { is finite for all } e \in E, \\
& \{e\} \in \text { Con for all } e \in E, \\
& Y \subseteq X \in \text { Con } \Longrightarrow Y \in \text { Con, and } \\
& X \in \text { Con } \& e \leq e^{\prime} \in X \Longrightarrow X \cup\{e\} \in \text { Con. }
\end{aligned}
$$

The configurations, $\mathcal{C}^{\infty}(E)$, of an event structure $E$ consist of those subsets $x \subseteq E$ which are

Consistent: $\forall X \subseteq x . X$ is finite $\Rightarrow X \in$ Con, and

Down-closed: $\forall e, e^{\prime} . e^{\prime} \leq e \in x \Longrightarrow e^{\prime} \in x$.

Often we shall be concerned with just the finite configurations of an event structure. We write $\mathcal{C}(E)$ for the finite configurations of an event structure $E$.

Two events which are both consistent and incomparable w.r.t. causal dependency in an event structure are regarded as concurrent. In games the relation of immediate dependency $e \rightarrow e^{\prime}$, meaning $e$ and $e^{\prime}$ are distinct with $e \leq e^{\prime}$ and no event in between, will play a very important role. For $X \subseteq E$ we write $[X]$ for $\left\{e \in E \mid \exists e^{\prime} \in X . e \leq e^{\prime}\right\}$, the down-closure of $X$; note if $X \in$ Con, then $[X] \in$ Con.

Example 1. The diagram below represents an event structure with five events in which, for example, $d$ causally depends on the previous occurrence of $a$ and $b$, while the two events $b$ and $c$ are inconsistent with each other (the squiggly line represents that the $\{b, c\}$ is not consistent).

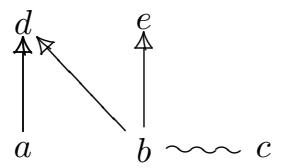


As $\{b, c\}$ is not consistent neither is $\{e, c\}$, but we need not draw this as it is entailed by the axioms on the consistency relation. Often consistency/inconsistency is determined in a binary fashion and we can take advantage of this in a diagram of the event structure. However, this is not always the case. Consider for instance the event structure consisting of the three events $1,2,3$ with the discrete order and consistency relation

$$
\text { Con }=\{\varnothing,\{1\},\{2\},\{3\},\{1,2\},\{1,3\},\{2,3\}\}
$$

Operations such as synchronized parallel composition are awkward to define directly on the simple event structures above. It is useful to broaden event structures to stable families, where operations are often carried out more easily, and then turned into event structures by the operation Pr below.

A stable family comprises $\mathcal{F}$, a nonempty family of finite subsets, called configurations, which satisfy:

Completeness: $\forall Z \subseteq \mathcal{F} . Z \uparrow \Longrightarrow \cup Z \in \mathcal{F}$;

Coincidence-freeness: For all $x \in \mathcal{F}, e, e^{\prime} \in x$ with $e \neq e^{\prime}$,

$$
\exists y \in \mathcal{F} . y \subseteq x \&\left(e \in y \Longleftrightarrow e^{\prime} \notin y\right)
$$

Stability: $\forall x, y \in \mathcal{F} . x \uparrow y \Longrightarrow x \cap y \in \mathcal{F}$.

Above, $Z \uparrow$ means $\exists x \in \mathcal{F} \forall z \in Z$. $z \subseteq x$, and expresses the compatibility of $Z$ in $\mathcal{F}$; we use $x \uparrow y$ for $\{x, y\} \uparrow$. We call elements of $\cup \mathcal{F}$ events of $\mathcal{F}$.

Proposition 1. Let $x$ be a configuration of a stable family $\mathcal{F}$. For $e, e^{\prime} \in x$ define

$$
e^{\prime} \leq_{x} \text { e iff } \forall y \in \mathcal{F} . y \subseteq x \quad \& e \in y \Longrightarrow e^{\prime} \in y .
$$

When $e \in x$ define the prime configuration

$$
[e]_{x}=\bigcap\{y \in \mathcal{F} \mid y \subseteq x \& e \in y\} .
$$

Then $\leq_{x}$ is a partial order and $[e]_{x}$ is a configuration such that

$$
[e]_{x}=\left\{e^{\prime} \in x \mid e^{\prime} \leq_{x} e\right\} .
$$

Moreover the configurations $y \subseteq x$ are exactly the down-closed subsets of $\leq_{x}$.

Proposition 2. Let $\mathcal{F}$ be a stable family. Then, $\operatorname{Pr}(\mathcal{F})=_{\text {def }}(P$, Con, $\leq)$ is an event structure where:

$$
\begin{aligned}
& P=\left\{[e]_{x} \mid e \in x \& x \in \mathcal{F}\right\}, \\
& Z \in \text { Con iff } Z \subseteq P \& \bigcup Z \in \mathcal{F} \text { and, } \\
& p \leq p^{\prime} \text { iff } p, p^{\prime} \in P \& p \subseteq p^{\prime} .
\end{aligned}
$$


A (partial) map of stable families $f: \mathcal{F} \rightarrow \mathcal{G}$ is a partial function $f$ from the events of $\mathcal{F}$ to the events of $\mathcal{G}$ such that for all configurations $x \in \mathcal{F}$,

$$
f x \in \mathcal{G} \&\left(\forall e_{1}, e_{2} \in x . f\left(e_{1}\right)=f\left(e_{2}\right) \Longrightarrow e_{1}=e_{2}\right) .
$$

Maps of event structures are maps of their stable families of configurations. Maps compose as functions. We say a map is total when it is total as a function.

Pr is the right adjoint of the "inclusion" functor, taking an event structure $E$ to the stable family $\mathcal{C}(E)$. The unit of the adjunction $E \rightarrow \operatorname{Pr}(\mathcal{C}(E))$ takes an event $e$ to the prime configuration $[e]=_{\text {def }}\left\{e^{\prime} \in E \mid e^{\prime} \leq e\right\}$. The counit max: $\mathcal{C}(\operatorname{Pr}(\mathcal{F})) \rightarrow \mathcal{F}$ takes prime configuration $[e]_{x}$ to its maximum event $e$; the image of a configuration $x \in \mathcal{C}(\operatorname{Pr}(\mathcal{F}))$ under the map max is $\bigcup x \in \mathcal{F}$.

Definition 1. Let $\mathcal{F}$ be a stable family. We use $x-\subset y$ to mean $y$ covers $x$ in $\mathcal{F}$, i.e. $x \subset y$ in $\mathcal{F}$ with nothing in between, and $x \stackrel{e}{e} \subset y$ to mean $x \cup\{e\}=y$ for $x, y \in \mathcal{F}$ and event $e \notin x$. We sometimes use $x \stackrel{e}{-}$, expressing that event $e$ is enabled at configuration $x$, when $x \stackrel{e}{-} \subset y$ for some $y$. W.r.t. $x \in \mathcal{F}$, write $[e)_{x}=d_{\operatorname{def}}\left\{e^{\prime} \in E \mid e^{\prime} \leq_{x} e \& e^{\prime} \neq e\right\}$, so, for example, $[e)_{x} \stackrel{e}{\subset} \subset[e]_{x}$. The relation of immediate dependence of event structures generalizes: with respect to $x \in \mathcal{F}$, the relation $e \rightarrow_{x} e^{\prime}$ means $e \leq_{x} e^{\prime}$ with $e \neq e^{\prime}$ and no event in between.

\section{Process operations}

\subsection{Products}

Let $\mathcal{A}$ and $\mathcal{B}$ be stable families with events $A$ and $B$, respectively. Their product, the stable family $\mathcal{A} \times \mathcal{B}$, has events comprising pairs in $A \times_{*} B={ }_{\text {def }}\{(a, *) \mid a \in A\} \cup$ $\{(a, b) \mid a \in A \& b \in B\} \cup\{(*, b) \mid b \in B\}$, the product of sets with partial functions, with (partial) projections $\pi_{1}$ and $\pi_{2}$-treating * as 'undefined'-with configurations $x \in \mathcal{A} \times \mathcal{B}$ iff

$$
\begin{aligned}
& x \text { is a finite subset of } A \times_{*} B \text { s.t. } \pi_{1} x \in \mathcal{A} \& \pi_{2} x \in \mathcal{B}, \\
& \forall e, e^{\prime} \in x . \pi_{1}(e)=\pi_{1}\left(e^{\prime}\right) \text { or } \pi_{2}(e)=\pi_{2}\left(e^{\prime}\right) \Rightarrow e=e^{\prime}, \& \\
& \forall e, e^{\prime} \in x . e \neq e^{\prime} \Rightarrow \exists y \subseteq x . \pi_{1} y \in \mathcal{A} \& \pi_{2} y \in \mathcal{B} \&\left(e \in y \Longleftrightarrow e^{\prime} \notin y\right) .
\end{aligned}
$$

Right adjoints preserve products. Consequently we obtain a product of event structures $A$ and $B$ by first regarding them as stable families $\mathcal{C}(A)$ and $\mathcal{C}(B)$, forming their product $\mathcal{C}(A) \times \mathcal{C}(B), \pi_{1}, \pi_{2}$, and then constructing the event structure

$$
A \times B={ }_{\text {def }} \operatorname{Pr}(\mathcal{C}(A) \times \mathcal{C}(B))
$$

and its projections as $\Pi_{1}={ }_{\text {def }} \pi_{1} \max$ and $\Pi_{2}==_{\text {def }} \pi_{2} \max$.

Later we shall use the following lemma relating immediate causal dependency in a product of stable families to immediate dependency in the components.

Lemma 1. Suppose $e \rightarrow_{x} e^{\prime}$ in a product of stable families $\mathcal{A} \times \mathcal{B}, \pi_{1}, \pi_{2}$.

(i) If $e=(a, *)$ then $e^{\prime}=\left(a^{\prime}, b\right)$ or $e^{\prime}=\left(a^{\prime}, *\right)$ with $a \rightarrow \pi_{\pi_{1} x} a^{\prime}$ in $\mathcal{A}$.

(ii) If $e^{\prime}=\left(a^{\prime}, *\right)$ then $e=(a, b)$ or $e=(a, *)$ with $a \rightarrow_{\pi_{1} x} a^{\prime}$ in $\mathcal{A}$.

(iii) If $e=(a, b)$ and $e^{\prime}=\left(a^{\prime}, b^{\prime}\right)$ then $a \rightarrow_{\pi_{1} x} a^{\prime}$ in $\mathcal{A}$ or $b \rightarrow_{\pi_{2} x} b^{\prime}$ in $\mathcal{B}$. 


\subsection{Restriction}

The restriction of $\mathcal{F}$ to a subset of events $R$ is the stable family $\mathcal{F} \uparrow R={ }_{\text {def }}$ $\{x \in \mathcal{F} \mid x \subseteq R\}$. Defining $E \uparrow R$, the restriction of an event structure $E$ to a subset of events $R$, to have events $E^{\prime}=\{e \in E \mid[e] \subseteq R\}$ with causal dependency and consistency induced by $E$, we obtain $\mathcal{C}(E \uparrow R)=\mathcal{C}(E) \uparrow R$.

Proposition 3. Let $\mathcal{F}$ be a stable family and $R$ a subset of its events. Then, $\operatorname{Pr}(\mathcal{F} \uparrow R)=\operatorname{Pr}(\mathcal{F}) \uparrow \max ^{-1} R$.

\subsection{Synchronized compositions}

Synchronized parallel compositions are obtained as restrictions of products to those events which are allowed to synchronize or occur asynchronously according to the specific synchronized composition. For example, the synchronized composition of Milner's CCS on stable families $\mathcal{A}$ and $\mathcal{B}$ (with labelled events) is defined as $\mathcal{A} \times \mathcal{B} \uparrow R$ where $R$ comprises events which are pairs $(a, *),(*, b)$ and $(a, b)$, where in the latter case the events $a$ of $\mathcal{A}$ and $b$ of $\mathcal{B}$ carry complementary labels. Similarly, synchronized compositions of event structures $A$ and $B$ are obtained as restrictions $A \times B \nmid R$. By Proposition 3, we can equivalently form a synchronized composition of event structures by forming the synchronized composition of their stable families of configurations, and then obtaining the resulting event structure - this has the advantage of eliminating superfluous events earlier.

\subsection{Projection}

Event structures support a simple form of hiding. Let $(E, \leq$, Con $)$ be an event structure. Let $V \subseteq E$ be a subset of 'visible' events. Define the projection of $E$ on $V$, to be $E \downarrow V={ }_{\text {def }}\left(V, \leq_{V}, \operatorname{Con}_{V}\right)$, where $v \leq_{V} v^{\prime}$ iff $v \leq v^{\prime} \& v, v^{\prime} \in V$ and $X \in \operatorname{Con}_{V}$ iff $X \in \operatorname{Con} \& X \subseteq V$.

\section{Event structures with polarities}

We shall represent both a game and a strategy in a game as an event structure with polarity, which comprises $(E, p o l)$ where $E$ is an event structure with a polarity function pol $: E \rightarrow\{+,-\}$ ascribing a polarity + (Player) or - (Opponent) to its events. The events correspond to (occurrences of) moves. Maps of event structures with polarity are maps of event structures which preserve polarity.

\subsection{Operations}

Dual The dual, $E^{\perp}$, of an event structure with polarity $E$ comprises a copy of the event structure $E$ but with a reversal of polarities. It obviously extends to a functor. Write $\bar{e} \in E^{\perp}$ for the event complementary to $e \in E$ and vice versa. 
Simple parallel composition This operation simply juxtaposes two event structures with polarity. Let $\left(A, \leq_{A}, \operatorname{Con}_{A}, \operatorname{pol}_{A}\right)$ and $\left(B, \leq_{B}, \operatorname{Con}_{B}, \operatorname{pol}_{B}\right)$ be event structures with polarity. The events of $A \| B$ are $(\{1\} \times A) \cup(\{2\} \times B)$, their polarities unchanged, with: the only relations of causal dependency given by $(1, a) \leq\left(1, a^{\prime}\right)$ iff $a \leq_{A} a^{\prime}$ and $(2, b) \leq\left(2, b^{\prime}\right)$ iff $b \leq_{B} b^{\prime}$; a subset of events $C$ is consistent in $A \| B$ iff $\{a \mid(1, a) \in C\} \in \operatorname{Con}_{A}$ and $\{b \mid(2, b) \in C\} \in \operatorname{Con}_{B}$. The operation extends to a functor - put the two maps in parallel. The empty event structure with polarity, written $\varnothing$, is the unit w.r.t. $\|$.

\section{Pre-strategies}

Let $A$ be an event structure with polarity, thought of as a game; its events stand for the possible occurrences of moves of Player and Opponent and its causal dependency and consistency relations for the constraints imposed by the game. A pre-strategy in $A$ is a total map $\sigma: S \rightarrow A$ from an event structure with polarity $S$. A pre-strategy represents a nondeterministic play of the game - all its moves are moves allowed by the game and obey the constraints of the game; the concept will later be refined to that of strategy (and winning strategy in Section 7). Two pre-strategies $\sigma: S \rightarrow A$ and $\tau: T \rightarrow A$ in $A$ will be essentially the same when they are isomorphic, i.e. there is an isomorphism $S \cong T$ such that

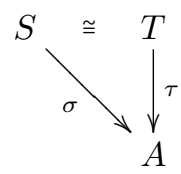

commutes. Then we write $\sigma \cong \tau$.

Let $A$ and $B$ be event structures with polarity. Following Joyal [13], a prestrategy from $A$ to $B$ is a pre-strategy in $A^{\perp} \| B$, so a total map $\sigma: S \rightarrow A^{\perp} \| B$. It thus determines a span

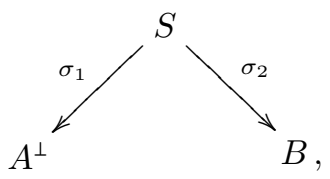

of event structures with polarity where $\sigma_{1}, \sigma_{2}$ are partial maps. In fact, a prestrategy from $A$ to $B$ corresponds to such spans where for all $s \in S$ either, but not both, $\sigma_{1}(s)$ or $\sigma_{2}(s)$ is defined. Two pre-strategies from $A$ to $B$ will be isomorphic when they are isomorphic as pre-strategies in $A^{\perp} \| B$, or equivalently are isomorphic as spans. We write $\sigma: A \nrightarrow B$ to express that $\sigma$ is a pre-strategy from $A$ to $B$. Note a pre-strategy $\sigma$ in a game $A$ coincides with a pre-strategy from the empty game $\sigma: \varnothing \rightarrow \rightarrow A$. 


\subsection{Composing pre-strategies}

Consider two pre-strategies $\sigma: A \nrightarrow B$ and $\tau: B \nrightarrow C$ as spans:
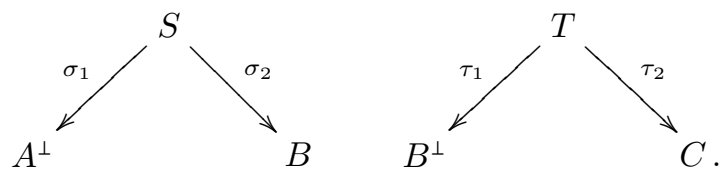

We show how to define their composition $\tau \odot \sigma: A \rightarrow C$ as the result of a synchronized composition, followed by projection to hide internal synchronization events. We first form the synchronized composition of $S$ and $T$ by restricting the product $S \times T$, with projections $\Pi_{1}: S \times T \rightarrow S$ and $\Pi_{2}: S \times T \rightarrow T$, to allow only those synchronizations associated with complementary events, of different polarities, in $B$ and $B^{\perp}$. Specifically, the synchronized composition is $S \times T \uparrow S y n$ where

$$
\begin{aligned}
\text { Syn }= & \left\{p \in S \times T \mid \sigma_{1} \Pi_{1}(p) \text { is defined } \& \Pi_{2}(p) \text { is undefined }\right\} \cup \\
& \left\{p \in S \times T \mid \tau_{2} \Pi_{2}(p) \text { is defined \& } \Pi_{1}(p) \text { is undefined }\right\} \cup \\
& \left\{p \in S \times T \mid \sigma_{2} \Pi_{1}(p)=\overline{\tau_{1} \Pi_{2}(p)} \text { with both defined }\right\} .
\end{aligned}
$$

We define $T \odot S==_{\text {def }}(S \times T \uparrow S y n) \downarrow V$ where

$V=\left\{p \in S \times T \uparrow S y n \mid \sigma_{1} \Pi_{1}(p)\right.$ is defined $\} \cup\left\{p \in S \times T \uparrow S y n \mid \tau_{2} \Pi_{2}(p)\right.$ is defined $\}$.

Finally, the composition $\tau \odot \sigma$ is defined to be the span

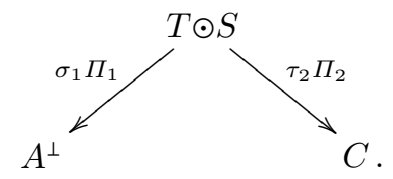

As remarked in Section 3.3, the same construction is achieved by first forming the synchronized composition of the stable families $\mathcal{C}(S)$ and $\mathcal{C}(T)$ (we often use this description in proofs):

Proposition 4. The composition $T \odot S=\operatorname{Pr}(\mathcal{C}(S) \times \mathcal{C}(T) \uparrow R) \downarrow V$, where

$$
\begin{aligned}
R= & \left\{(s, *) \mid s \in S \& \sigma_{1}(s) \text { is defined }\right\} \cup\left\{(*, t) \mid t \in T \& \tau_{2}(t) \text { is defined }\right\} \cup \\
& \left\{(s, t) \mid s \in S \& t \in T \& \sigma_{2}(s)=\overline{\tau_{1}(t)} \text { with both defined }\right\} .
\end{aligned}
$$

The span $\tau \odot \sigma$ comprises maps $v_{1}: T \odot S \rightarrow A^{\perp}$ and $v_{2}: T \odot S \rightarrow C$, which on events $p$ of $T \odot S$ act so $v_{1}(p)=\sigma_{1}(s)$ when $\max (p)=(s, *)$ and $v_{2}(p)=\tau_{2}(t)$ when $\max (p)=(*, t)$, and are undefined elsewhere.

The natural isomorphism $S \times(T \times U) \cong(S \times T) \times U$, associated with the product of event structures $S, T, U$, restricts to the required isomorphism of spans as the synchronizations involved in successive compositions are disjoint:

Proposition 5. Let $\sigma: A \nrightarrow B, \tau: B \nrightarrow C$ and $v: C \nrightarrow D$ be pre-strategies. The two compositions $v \odot(\tau \odot \sigma)$ and $(v \odot \tau) \odot \sigma$ are isomorphic. 


\subsection{Concurrent copy-cat}

Identities w.r.t. composition are given by copy-cat strategies. Let $A$ be an event structure with polarity. The copy-cat strategy from $A$ to $A$ is an instance of a pre-strategy, so a total map $\gamma_{A}: \mathbb{C C}_{A} \rightarrow A^{\perp} \| A$. It describes a concurrent, or distributed, strategy based on the idea that Player moves, of +ve polarity, always copy previous corresponding moves of Opponent, of -ve polarity.

For $c \in A^{\perp} \| A$ we use $\bar{c}$ to mean the corresponding copy of $c$, of opposite polarity, in the alternative component, i.e. $\overline{(1, a)}=(2, \bar{a})$ and $\overline{(2, a)}=(1, \bar{a})$. Define $\mathbb{C C}_{A}$ to comprise the event structure with polarity $A^{\perp} \| A$ together with extra causal dependencies $\bar{c} \leq_{\mathrm{CC}_{A}} c$ for all events $c$ with $p^{\circ} l_{A^{\perp} \| A}(c)=+$.

Proposition 6. Let $A$ be an event structure with polarity. Then $\mathrm{CC}_{A}$ is an event structure with polarity. Moreover,

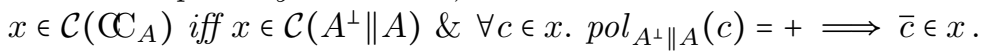

The copy-cat pre-strategy $\gamma_{A}: A \rightarrow A$ is defined to be the map $\gamma_{A}: \mathbb{C C}_{A} \rightarrow$ $A^{\perp} \| A$ where $\gamma_{A}$ is the identity on the common set of events.

Example 2. We illustrate the construction of the copy-cat strategy for the event structure $A$ comprising the single immediate dependency $a_{1} \rightarrow a_{2}$ from an Opponent move $a_{1}$ to a Player move $a_{2}$. The event structure $\mathrm{CC}_{A}$ is obtained from $A^{\perp} \| A$ by adjoining the additional immediate dependencies shown:

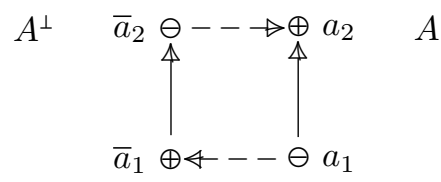

The pre-strategy $\gamma_{A}: \mathbb{C C}_{A} \rightarrow A^{\perp} \| A$ is defined to act simply as the identity function on events.

\section{Strategies}

The main result of [5], presented summarily here, is that two conditions on prestrategies, receptivity and innocence, are necessary and sufficient for copy-cat to behave as identity w.r.t. the composition of pre-strategies. Receptivity ensures an openness to all possible moves of Opponent. Innocence restricts the behaviour of Player; Player may only introduce new relations of immediate causality of the form $\ominus \rightarrow \oplus$ beyond those imposed by the game.

Receptivity. A pre-strategy $\sigma$ is receptive iff $\sigma x \stackrel{a}{\complement} \& p o l_{A}(a)=-\Rightarrow \exists ! s \in$ S. $x \stackrel{s}{\subset} \& \sigma(s)=a$.

Innocence. A pre-strategy $\sigma$ is innocent when it is both + -innocent: if $s \rightarrow s^{\prime} \& \operatorname{pol}(s)=+$ then $\sigma(s) \rightarrow \sigma\left(s^{\prime}\right)$, and - -innocent: if $s \rightarrow s^{\prime} \& \operatorname{pol}\left(s^{\prime}\right)=-$ then $\sigma(s) \rightarrow \sigma\left(s^{\prime}\right)$. 
Theorem 1. Let $\sigma: A \rightarrow B$ be pre-strategy. Copy-cat behaves as identity w.r.t. composition, i.e. $\sigma \circ \gamma_{A} \cong \sigma$ and $\gamma_{B} \circ \sigma \cong \sigma$, iff $\sigma$ is receptive and innocent. Copy-cat pre-stategies $\gamma_{A}: A \rightarrow A$ are receptive and innocent.

\subsection{The bicategory of concurrent games and strategies}

Theorem 1 motivates the definition of a strategy as a pre-strategy which is receptive and innocent. In fact, we obtain a bicategory, Games, in which the objects are event structures with polarity - the games, the arrows from $A$ to $B$ are strategies $\sigma: A \nrightarrow B$ and the 2-cells are maps of spans. The vertical composition of 2 -cells is the usual composition of maps of spans. Horizontal composition is given by the composition of strategies $\odot$ (which extends to a functor on 2-cells via the functoriality of synchronized composition).

A strategy $\sigma: A \rightarrow B$ corresponds to a dual strategy $\sigma^{\perp}: B^{\perp} \rightarrow A^{\perp}$. This duality arises from the correspondence

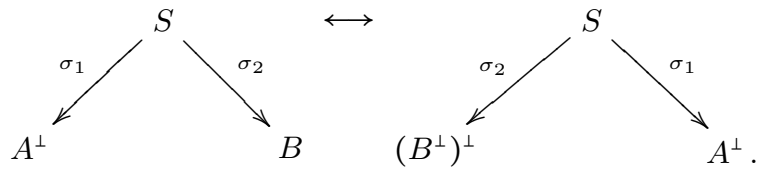

The dual of copy-cat, $\gamma_{A}^{\perp}$, is isomorphic to the copy-cat of the dual, $\gamma_{A^{\perp}}$, for $A$ an event structure with polarity. The dual of a composition of pre-strategies $(\tau \odot \sigma)^{\perp}$ is isomorphic to the composition $\sigma^{\perp} \odot \tau^{\perp}$. This duality will be maintained in all the bicategories of games we shall consider.

\subsection{The subcategory of deterministic strategies}

Say an event structure with polarity $S$ is deterministic iff

$$
\forall X \subseteq_{\text {fin }} S . N e g[X] \in \operatorname{Con}_{S} \Longrightarrow X \in \operatorname{Con}_{S},
$$

where $N e g[X]=$ def $\left\{s^{\prime} \in S \mid \operatorname{pol}\left(s^{\prime}\right)=-\& \exists s \in X . s^{\prime} \leq s\right\}$. In other words, $S$ is deterministic iff any finite set of moves is consistent when it causally depends only on a consistent set of opponent moves. Say a strategy $\sigma: S \rightarrow A$ is deterministic if $S$ is deterministic.

Lemma 2. An event structure with polarity $S$ is deterministic iff

$$
\forall s, s^{\prime} \in S, x \in \mathcal{C}(S) . \quad x \stackrel{s}{\subset} \& x \stackrel{s^{\prime}}{\subset} \& \operatorname{pol}(s)=+\Longrightarrow x \cup\left\{s, s^{\prime}\right\} \in \mathcal{C}(S) .
$$

In general, a copy-cat strategy can fail to be deterministic, illustrated below.

Example 3. Take $A$ to consist of two events, one +ve and one -ve event, inconsistent with each other. The construction $\mathrm{CC}_{A}$ :

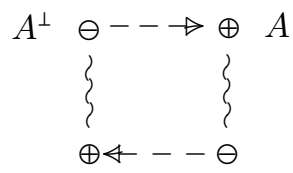


To see $\mathbb{C C}_{A}$ is not deterministic, take $x$ to be the singleton set consisting e.g. of the -ve event on the left and $s, s^{\prime}$ to be the +ve and -ve events on the right.

Copy-cat $\gamma_{A}$ is deterministic iff immediate conflict in $A$ respects polarity, or equivalently that there is no immediate conflict between +ve and -ve events, a condition we call 'race-free.'

Lemma 3. Let $A$ be an event structure with polarity. The copy-cat strategy $\gamma_{A}$ is deterministic iff

$$
\begin{aligned}
\forall x \in \mathcal{C}(A) . x \stackrel{a}{ } \subset \& x \stackrel{a^{\prime}}{\longrightarrow} \& \operatorname{pol}(a) & =+\& \operatorname{pol}\left(a^{\prime}\right)=- & \\
& \Longrightarrow x \cup\left\{a, a^{\prime}\right\} \in \mathcal{C}(A) . &
\end{aligned}
$$

Lemma 4. The composition of deterministic strategies is deterministic.

Lemma 5. A deterministic strategy $\sigma: S \rightarrow A$ is injective on configurations (equivalently, $\sigma$ is mono in the category of event structures with polarity).

We obtain a sub-bicategory DGames of Games by restricting objects to race-free games and strategies to being deterministic. Via Lemma 5, deterministic strategies in a game correspond to certain subfamilies of configurations of the game. A characterization of those subfamilies which correspond to deterministic strategies shows them to coincide with the receptive ingenuous strategies of Mimram and Melliès [9]. Via the presentation of deterministic strategies as families DGames is equivalent to an order-enriched category.

\section{Winning, losing and drawing}

A game with winning/losing conditions comprises $G=(A, W, L)$ where $A$ is an event structure with polarity and $W \subseteq \mathcal{C}^{\infty}(A)$ consists of the winning configurations disjoint from the losing configurations $L \subseteq \mathcal{C}^{\infty}(A)$ for Player. We do not insist that $W$ and $L$ partition the set $\mathcal{C}^{\infty}(A)$ - there may be neutral configurations at which Player and Opponent draw.

A strategy in $G$ is a strategy in $A$. A strategy in $G$ is regarded as winning if it always prescribes Player moves to end up in a winning configuration, no matter what the activity or inactivity of Opponent. Formally, a strategy $\sigma: S \rightarrow A$ in $G$ is winning (for Player) if $\sigma x \in W$ for all +-maximal configurations $x \in \mathcal{C}^{\infty}(S)$ a configuration $x$ is +-maximal if whenever $x \stackrel{s}{\subset}$ then the event $s$ has -ve polarity. Any achievable position $z \in \mathcal{C}^{\infty}(S)$ of the game can be extended to a +maximal, so winning, configuration (via Zorn's Lemma). So a strategy prescribes Player moves to reach a winning configuration whatever state of play is achieved following the strategy. Note that for a game $A$, if winning conditions $W=\mathcal{C}^{\infty}(A)$, i.e. every configuration is winning, then any strategy in $A$ is a winning strategy.

Informally, we can also understand a strategy as winning for Player if when played against any counter-strategy of Opponent, the final result is a win for Player. Suppose $\sigma: S \rightarrow A$ is a strategy in a game $(A, W)$. A counter-strategy is 
strategy of Opponent, so a strategy $\tau: T \rightarrow A^{\perp}$ in the dual game. We can view $\sigma$ as a strategy $\sigma: \varnothing \rightarrow A$ and $\tau$ as a strategy $\tau: A \nrightarrow \varnothing$. Their composition $\tau \odot \sigma: \varnothing \rightarrow 1>\varnothing$ is not in itself so informative. Rather it is the status of the configurations in $\mathcal{C}^{\infty}(A)$ their full interaction induces which decides which of Player or Opponent wins. For this we should consider the composition of $\sigma$ and $\tau$ before hiding internal synchronizations:

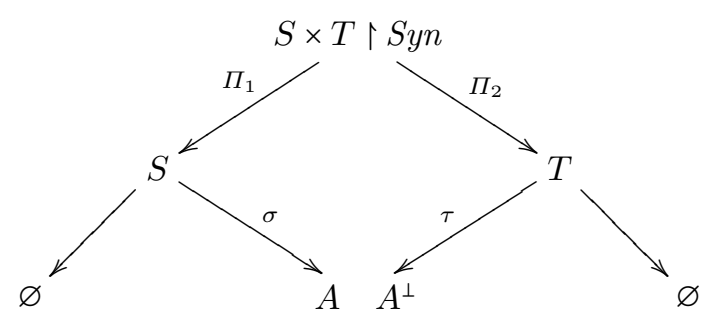

where

$$
\text { Syn }=\left\{p \in S \times T \mid \sigma \Pi_{1}(p)=\overline{\tau \Pi_{2}(p)} \text { with both defined }\right\} .
$$

Because $\sigma$ or $\tau$ may be nondeterministic there can be more than one maximal configuration $z$ in $\mathcal{C}^{\infty}(S \times T \uparrow S y n)$. A maximal configuration $z$ in $\mathcal{C}^{\infty}(S \times T \uparrow S y n)$ images to a configuration $\sigma \Pi_{1} z=\tau \Pi_{2} z$ in $\mathcal{C}^{\infty}(A)$. Define the set of results of the interaction of $\sigma$ and $\tau$ to be

$$
\langle\sigma, \tau\rangle=_{\operatorname{def}}\left\{\sigma \Pi_{1} z \mid z \text { is maximal in } \mathcal{C}^{\infty}(S \times T \uparrow S y n)\right\}
$$

A configuration $x \in\langle\sigma, \tau\rangle$, resulting from a play of $\sigma$ against $\tau$ may be a win for Player, if $x \in W$, a loss for Player and a win for Opponent, if $x \in L$, or a draw, when $x \notin W \cup L$.

Lemma 6. Let $\sigma: S \rightarrow A$ be a strategy in a game $(A, W, L)$. The strategy $\sigma$ is a winning for Player iff $\langle\sigma, \tau\rangle \subseteq W$ for all (deterministic) strategies $\tau: T \rightarrow A^{\perp}$.

Corollary 1. There are the following three equivalent ways to say that a strategy $\sigma: S \rightarrow A$ is winning in $(A, W, L)$ :

1. $\sigma x \in W$ for all +-maximal configurations $x \in \mathcal{C}^{\infty}(S)$, i.e. the strategy prescribes Player moves to reach a winning configuration, no matter what the activity or inactivity of Opponent;

2. $\langle\sigma, \tau\rangle \subseteq W$ for all strategies $\tau: T \rightarrow A^{\perp}$, i.e. all plays against counterstrategies of the Opponent result in a win for Player;

3. $\langle\sigma, \tau\rangle \subseteq W$ for all deterministic strategies $\tau: T \rightarrow A^{\perp}$, i.e. all plays against deterministic counter-strategies of the Opponent result in a win for Player.

The proof of Lemma 6 relies on the following general lemma which will be useful later. Its proof and those of the lemma and corollary above can be found in [1] - they are essentially repeats of the corresponding proofs for games with just winning conditions. 
Lemma 7. Let $\sigma: S \rightarrow A^{\perp} \| B$ and $\tau: T \rightarrow B^{\perp} \| C$ be receptive pre-strategies. Then,

$$
\begin{aligned}
& z \in \mathcal{C}^{\infty}(T \times S \uparrow \text { Syn }) \text { is +-maximal iff } \\
& \Pi_{1} z \in \mathcal{C}^{\infty}(S) \text { is }+ \text {-maximal \& } \Pi_{2} z \in \mathcal{C}^{\infty}(T) \text { is t-maximal. }
\end{aligned}
$$

A convention is being adopted in Lemma 7. The events of $T \times S \uparrow S y n$ are constructed as prime configurations $p$ of a stable family, and as such $\max (p)$ has the form $(s, *),(*, t)$ or $(s, t)$, with $s \in S$ and $t \in T$. An event $p$ with $\max (p)$ of the form $(s, *)$ or $(*, t)$ adopts the polarity of the event $s$ or $t$, while those $p$ with $\max (p)=(s, t)$ are regarded as not having a polarity. By $x \in \mathcal{C}^{\infty}(T \times S \uparrow S y n)$ is + -maximal is meant that whenever $x \stackrel{e}{-}$ the event $e$ has - ve polarity.

\section{Operations}

\subsection{Dual}

There is an obvious dual of a game $G=\left(A, W_{G}, L_{G}\right)$ which reverses the role of Player and Opponent: $G^{\perp}=\left(A^{\perp}, W_{G^{\perp}}, L_{G^{\perp}}\right)$ where

$$
\begin{aligned}
x \in W_{G^{\perp}} & \Longleftrightarrow \bar{x} \in L_{G} \text { and } \\
x \in L_{G^{\perp}} & \Longleftrightarrow \bar{x} \in W_{G} .
\end{aligned}
$$

Here, and in future, we extend the bar-notation for the bijection between events of $A$ and $A^{\perp}$ to configurations: a configuration $x \in \mathcal{C}^{\infty}(A)$ corresponds to a configuration $\bar{x}=$ def $\{\bar{a} \mid a \in x\} \in \mathcal{C}^{\infty}\left(A^{\perp}\right)$.

\subsection{Parallel composition}

The parallel composition of two games $G=\left(A, W_{G}, L_{G}\right), H=\left(B, W_{H}, L_{H}\right)$ is

$$
G \| H=_{\operatorname{def}}\left(A\left\|B, W_{G}\right\| \mathcal{C}^{\infty}(B) \cup \mathcal{C}^{\infty}(A)\left\|W_{H}, L_{G}\right\| L_{H}\right)
$$

where $X \| Y=\{\{1\} \times x \cup\{2\} \times y \mid x \in X \& y \in Y\}$ when $X$ and $Y$ are subsets of configurations. In other words, for $x \in \mathcal{C}^{\infty}(A \| B)$,

$$
\begin{aligned}
x \in W_{G \| H} & \Longleftrightarrow x_{1} \in W_{G} \text { or } x_{2} \in W_{H}, \text { and } \\
x \in L_{G \| H} & \Longleftrightarrow x_{1} \in L_{G} \& x_{2} \in L_{H},
\end{aligned}
$$

where $x_{1}=\{a \mid(1, a) \in x\}$ and $x_{2}=\{b \mid(2, b) \in x\}$. To win in $G \| H$ is to win in either game; to lose is to lose in both games. The unit of $\|$ is $(\varnothing, \varnothing,\{\varnothing\})$.

\subsection{Tensor}

For games $G=\left(A, W_{G}, L_{G}\right), H=\left(B, W_{H}, L_{H}\right)$, defining $G \otimes H==_{\operatorname{def}}\left(G^{\perp} \| H^{\perp}\right)^{\perp}$ we obtain a game where to win is to win in both games $G$ and $H$, and to lose is to lose in either game. More explicitly,

$$
\left(A, W_{G}, L_{G}\right) \otimes\left(B, W_{H}, L_{H}\right)=_{\text {def }}\left(A\left\|B, W_{G}\right\| W_{H}, L_{G}\left\|\mathcal{C}^{\infty}(B) \cup \mathcal{C}^{\infty}(A)\right\| L_{H}\right) .
$$

The unit of $\otimes$ is $(\varnothing,\{\varnothing\}, \varnothing)$. 


\subsection{Function space}

With $G \multimap H=_{\text {def }} G^{\perp} \| H$ a win in $G \multimap H$ is a win in $H$ conditional on not losing in $G$ :

Proposition 1 Let $G=\left(A, W_{G}, L_{G}\right)$ and $H=\left(B, W_{H}, L_{H}\right)$ be games with winning conditions. Write $W_{G \rightarrow H}, L_{G \rightarrow H}$ for the winning, respectively losing conditions of $G \multimap H$. For $x \in \mathcal{C}^{\infty}\left(A^{\perp} \| B\right)$,

$$
\begin{aligned}
& x \in W_{G \rightarrow H} \quad \text { iff } \quad \overline{x_{1}} \notin L_{G} \Longrightarrow x_{2} \in W_{H} \text {, and } \\
& x \in L_{G \rightarrow H} \quad \text { iff } \quad x_{2} \notin L_{H} \Longrightarrow \overline{x_{1}} \in W_{G} \text {. }
\end{aligned}
$$

Proof. Letting $x \in \mathcal{C}^{\infty}\left(A^{\perp} \| B\right)$,

$$
\begin{aligned}
x \in W_{G \rightarrow H} & \text { iff } x \in W_{G^{\perp} \| H} \\
& \text { iff } x_{1} \in W_{G^{\perp}} \text { or } x_{2} \in W_{H} \\
& \text { iff } \overline{x_{1}} \in L_{G} \text { or } x_{2} \in W_{H} \\
& \text { iff } \overline{x_{1}} \notin L_{G} \Longrightarrow x_{2} \in W_{H} .
\end{aligned}
$$

The other part is proved similarly.

\section{The bicategory of winning strategies}

We can again follow Joyal and define strategies between games now with winning/losing conditions: a (winning) strategy from $G$, a game with winning/losing conditions, to another $H$ is a (winning) strategy in $G \rightarrow H=G^{\perp} \| H$. We compose strategies as before. We show that the composition of winning strategies is winning.

Lemma 8. Let $\sigma$ be a winning strategy in $G^{\perp} \| H$ and $\tau$ be a winning strategy in $H^{\perp} \| K$. Their composition $\tau \odot \sigma$ is a winning strategy in $G^{\perp} \| K$.

Proof. Suppose $x \in \mathcal{C}^{\infty}(T \odot S)$ is +-maximal. The event structure $T \odot S$ is obtained as the projection of $S \times T \uparrow S y n$ to the set of 'visible' events $V$. Hence the down-closure [x] in $S \times T \uparrow S y n$ forms a configuration $[x] \in \mathcal{C}^{\infty}(S \times T \uparrow S y n)$. By Zorn's Lemma we can extend $[x]$ to a maximal configuration $z \supseteq[x]$ in $\mathcal{C}^{\infty}(S \times T \uparrow S y n)$ with the property that all events of $z \backslash[x]$ are synchronizations of the form $p$ with $\max (p)=(s, t)$ for $s \in S$ and $t \in T$. Then, $z$ will be +-maximal in $\mathcal{C}^{\infty}(S \times T \uparrow S y n)$ with

$$
\sigma_{1} \Pi_{1} z=\sigma_{1} \Pi_{1}[x] \quad \& \quad \tau_{2} \Pi_{2} z=\tau_{2} \Pi_{2}[x] .
$$

By Lemma 7,

$$
\Pi_{1} z \text { is +-maximal in } S \quad \& \quad \Pi_{2} z \text { is +-maximal in } T .
$$

As $\sigma$ and $\tau$ are winning,

$$
\sigma \Pi_{1} z \in W_{G^{\perp} \| H} \quad \& \quad \tau \Pi_{2} z \in W_{H^{\perp} \| K} .
$$


Now $\sigma \Pi_{1} z \in W_{G^{\perp} \| H}$ expresses that

$$
\overline{\sigma_{1} \Pi_{1} z} \notin L_{G} \Longrightarrow \sigma_{2} \Pi_{1} z \in W_{H}
$$

and $\tau \Pi_{2} z \in W_{H^{\perp} \| K}$ that

$$
\overline{\tau_{1} \Pi_{2} z} \notin L_{H} \Longrightarrow \tau_{2} \Pi_{2} z \in W_{K},
$$

by Proposition 1. But

$$
\sigma_{2} \Pi_{1} z \in W_{H} \Longrightarrow \sigma_{2} \Pi_{1} z \notin L_{H}
$$

as $W_{H}$ and $L_{H}$ are disjoint. Moreover, $\sigma_{2} \Pi_{1} z=\overline{\tau_{1} \Pi_{2} z}$. So (2), (3) and (4) yield

$$
\overline{\sigma_{1} \Pi_{1} z} \notin L_{G} \Longrightarrow \tau_{2} \Pi_{2} z \in W_{K} .
$$

By (1)

$$
\overline{\sigma_{1} \Pi_{1}[x]} \notin L_{G} \Longrightarrow \tau_{2} \Pi_{2}[x] \in W_{K},
$$

i.e. from the definition of $\tau \odot \sigma$,

$$
\overline{(\tau \odot \sigma)_{1} x} \notin L_{G} \Longrightarrow(\tau \odot \sigma)_{2} x \in W_{K}
$$

in the span of the composition $\tau \odot \sigma$. Hence $\tau \odot \sigma x \in W_{G^{\perp} \| K}$ whenever $x$ is a +-maximal configuration of $T \odot S$, ensuring $\tau \odot \sigma$ is a winning strategy.

For a general game with winning/losing conditions $(A, W, L)$ the copy-cat strategy need not be winning:

Example 4. Let $A$ consist of two events, one +ve event $\oplus$ and one -ve event $\ominus$, inconsistent with each other. Take as winning conditions the set $W=\{\{\oplus\}\}$ and as losing conditions the set $L=\{\{\ominus\}\}$. The event structure $\mathbb{C C}_{A}$ :

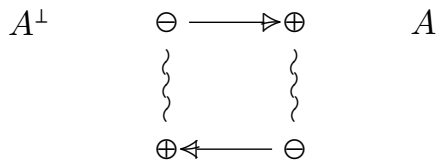

To see $\mathrm{CC}_{A}$ is not winning consider the configuration $x$ consisting of the two -ve events in $\mathbb{C C}_{A}$. Then $x$ is +-maximal as any +ve event is inconsistent with $x$. However, $\bar{x}_{1} \notin L$ while $x_{2} \notin W$, failing the winning conditions of $(A, W, L) \multimap$ $(A, W, L)$.

Each event structure with polarity $A$ possesses a 'Scott order' on its configurations $\mathcal{C}^{\infty}(A)$ :

$$
x^{\prime} \sqsubseteq x \text { iff } x^{\prime} \beth^{-} x \cap x^{\prime} \subseteq^{+} x .
$$

Above we use the special inclusions

$$
\begin{aligned}
& x \subseteq^{-} y \text { iff } x \subseteq y \& \operatorname{pol}_{A}(y \backslash x) \subseteq\{-\}, \text { and } \\
& x \subseteq^{+} y \text { iff } x \subseteq y \& \operatorname{pol}_{A}(y \backslash x) \subseteq\{+\}
\end{aligned}
$$


for $x, y \in \mathcal{C}^{\infty}(A)$. A necessary and sufficient for copy-cat to be winning w.r.t. a game $(A, W, L)$ :

$$
\begin{aligned}
\forall x, x^{\prime} \in \mathcal{C}^{\infty}(A) . & \text { if } x^{\prime} \sqsubseteq x \& x^{\prime} \text { is }+ \text {-maximal \& } x \text { is --maximal, } \\
& \text { then } x \in L \text { or } x^{\prime} \in W .
\end{aligned}
$$

(Cwins)

Lemma 9. Let $(A, W, L)$ be a game with winning/losing conditions. The copycat strategy $\gamma_{A}: \mathbb{C C}_{A} \rightarrow A^{\perp} \| A$ is winning iff $(A, W, L)$ satisfies (Cwins).

Proof. It can be shown that

$$
z \in \mathcal{C}^{\infty}\left(\mathcal{C C}_{A}\right) \text { iff } z=\{1\} \times \bar{x} \cup\{2\} \times x^{\prime} \text { with } x^{\prime} \sqsubseteq_{A} x,
$$

for $x, x^{\prime} \in \mathcal{C}^{\infty}(A)$ - see Lemma 54 in the Aarhus notes [1]. In this situation $z$ is +maximal iff both $x$ is --maximal and $x^{\prime}$ is +-maximal. Thus (Cwins) expresses precisely that copy-cat is winning in $(A, W, L) \multimap(A, W, L)$.

For race-free games we can simplify (Cwins), the condition for copy-cat to be winning. Copy-cat is a winning strategy for a race-free game iff no maximal configuration of the game is neutral.

Proposition 2 Assume $A$ is a race-free event structure with polarity. For a game $(A, W, L)$, the property (Cwins) holds iff $x \in W \cup L$ for all maximal configurations $x \in \mathcal{C}^{\infty}(A)$.

Proof. For $x, x^{\prime} \in \mathcal{C}^{\infty}(A)$, assume

$$
x^{\prime} \text { 다 \& } x^{\prime} \text { is +-maximal \& } x \text { is --maximal. }
$$

As $x^{\prime} \supseteq^{-} x \cap x^{\prime} \subseteq^{+} x$, there are covering chains associated with purely + ve and -ve events from $x \cap x^{\prime}$ to $x$ and $x^{\prime}$, respectively:

$$
\begin{aligned}
& x \cap x^{\prime} \stackrel{+}{\sqsubset} \cdots \stackrel{+}{\subset} x, \\
& x \cap x^{\prime} \stackrel{-}{\sqsubset} \cdots \stackrel{-}{\subset} x^{\prime} .
\end{aligned}
$$

If one of the covering chains is of zero length then so must the other be otherwise we contradict one or other of the maximality assumptions. On the other hand, if both are nonempty, by repeated use of (Race-free) we again contradict a maximality assumption, e.g.

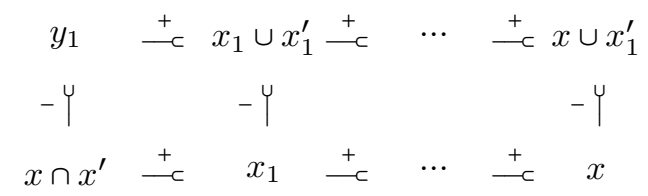

shows how a repeated use of (Race-free) contradicts the --maximality of $x$. We conclude that both covering chains must be of zero length, making $x=x \cap x^{\prime}=$ $x^{\prime}$. As configurations which are both + and --maximal are simply maximal, the property (Cwins) now expresses that all maximal configurations are either winning or losing. 
We can now refine the bicategory of strategies Games to the bicategory WLGames with objects $G, H, \cdots$, games with winning/losing conditions satisfying (Cwins), and arrows winning strategies $G \rightarrow H ; 2$-cells, their vertical and horizontal composition is as before. Via the constructions of Section 8, the bicategory is rich in categorical structure, and is in particular monoidal-closed. Its restriction to deterministic strategies yields a bicategory equivalent to a simpler order-enriched category.

\section{Games with imperfect information}

Consider the game "rock, scissors, paper" in which the two participants Player and Opponent independently sign one of $r$ ("rock"), $s$ ("scissors") or $p$ ("paper"). The participant with the dominant sign w.r.t. the relation

$$
r \text { beats } s, s \text { beats } p \text { and } p \text { beats } r
$$

wins. It seems sensible to represent this game by $R S P$, the event structure with polarity
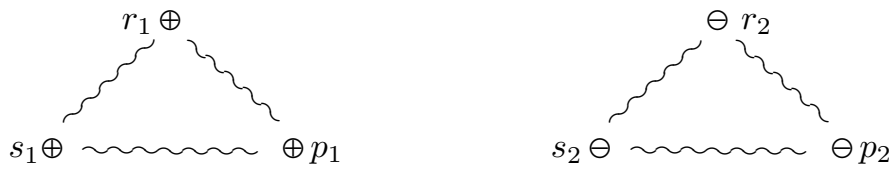

comprising the three mutually inconsistent possible signings of Player in parallel with the three mutually inconsistent signings of Opponent. What are the winning/losing conditions? A reasonable choice is to take the winning and losing configurations (for Player) to be given by

$$
W=\left\{\left\{r_{1}, s_{2}\right\},\left\{s_{1}, p_{2}\right\},\left\{p_{1}, r_{2}\right\}\right\} \text { and } L=\left\{\left\{s_{1}, r_{2}\right\},\left\{p_{1}, s_{2}\right\},\left\{r_{1}, p_{2}\right\}\right\} .
$$

All other configurations are neutral, neither winning nor losing.

In this game it turns out that no participant has a winning strategy, which agrees with our informal understanding of the game "rock, scissors, paper." However on closer inspection there is a mismatch between the possible strategies allowed in our idealised mathematical and those of the real game. To make the mismatch clearer, let us bias the game in favour of Player by making the empty configuration winning, i.e. now $\varnothing \in W$. In this case there is a winning strategy for Player, viz. await the move of Opponent and then beat it with a dominant move. Explicitly, the winning strategy $\sigma: S \rightarrow R S P$ is given as the obvious map from $S$, the following event structure with polarity:

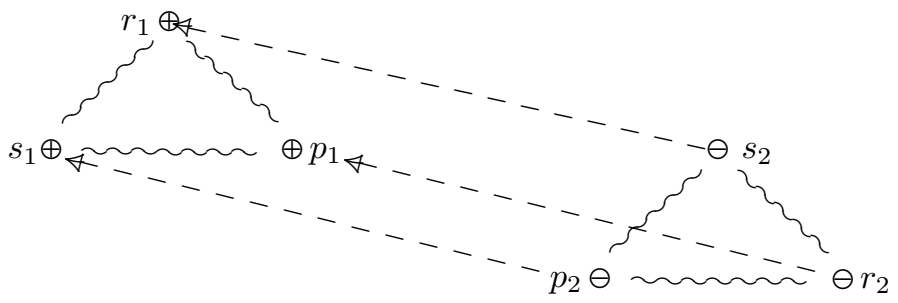


But this strategy hardly enters into the spirit of "rock, scissors, paper" where the participants are intended to make their moves independently. The problem with the game $R S P$ as it stands is that it is a game of perfect information in the sense that all moves are visible to both participants. This permits the winning strategy above with its unwanted dependencies on moves which should be unseen by Player. To adequately model "rock, scissors, paper" requires a game of imperfect information where some moves are masked, or inaccessible, and strategies with dependencies on unseen moves are ruled out.

We extend concurrent games to games with imperfect information. To do so in way that respects the operations of the bicategory of games we suppose a fixed preorder of levels $(\Lambda, \leq)$. The levels are to be thought of as levels of access, or permission. Moves in games and strategies are to respect levels: moves will be assigned levels in such a way that a move is only permitted to causally depend on moves at equal or lower levels; it is as if from a level only moves of equal or lower level can be seen.

An $\Lambda$-game $(G, l)$ comprises a game $G=(A, W, L)$ with winning/losing conditions together with a level function $l: A \rightarrow \Lambda$ such that

$$
a \leq_{A} a^{\prime} \Longrightarrow l(a) \leq l\left(a^{\prime}\right)
$$

for all $a, a^{\prime} \in A$. A $\Lambda$-strategy in the $\Lambda$-game $(G, l)$ is a strategy $\sigma: S \rightarrow A$ for which

$$
s \leq_{S} s^{\prime} \Longrightarrow l \sigma(s) \leq l \sigma\left(s^{\prime}\right)
$$

for all $s, s^{\prime} \in S$.

For example, for "rock, scissors, paper" we can take $\Lambda$ to be the discrete preorder consisting of levels 1 and 2 unrelated to each other under $\leq$. To make $R S P$ into a suitable $\Lambda$-game the level function $l$ takes +ve events in $R S P$ to level 1 and -ve events to level 2. The strategy above, where Player awaits the move of Opponent then beats it with a dominant move, is now disallowed because it is not a $\Lambda$-strategy - it introduces causal dependencies which do not respect levels. If instead we took $\Lambda$ to be the unique preorder on a single level the $\Lambda$-strategies would coincide with all the strategies.

Games with imperfect information are central to the semantics of Hintikka and Sandu's independence-friendly (IF) logic in which special quantifiers restrict those strategies permitted to establish an assertion [14]. A recent paper [15], building on a concurrent-game semantics of predicate calculus [6], proposes a compositional semantics for a variant of IF logic in which assertions of IF logic denote concurrent games with imperfect information.

\subsection{The bicategory of $\Lambda$-games}

The introduction of levels meshes smoothly with the bicategorical structure on games.

For a $\Lambda$-game $\left(G, l_{G}\right)$, define its dual $\left(G, l_{G}\right)^{\perp}$ to be $\left(G^{\perp}, l_{G^{\perp}}\right)$ where $l_{G^{\perp}}(\bar{a})=$ $l_{G}(a)$, for $a$ an event of $G$. 
For $\Lambda$-games $\left(G, l_{G}\right)$ and $\left(H, l_{H}\right)$, define their parallel composition $\left(G, l_{G}\right) \|\left(H, l_{H}\right)$ to be $\left(G \| H, l_{G \| H}\right)$ where $l_{G \| H}((1, a))=l_{G}(a)$, for $a$ an event of $G$, and $l_{G \| H}((2, b))=$ $l_{H}(b)$, for $b$ an event of $H$.

A strategy between $\Lambda$-games from $\left(G, l_{G}\right)$ to $\left(H, l_{H}\right)$ is a strategy in $\left(G, l_{G}\right)^{\perp} \|\left(H, l_{H}\right)$.

\section{Proposition 3}

(i) Let $\left(G, l_{G}\right)$ be a $\Lambda$-game where $G$ satisfies (Cwins). The copy-cat strategy on $G$ is a $\Lambda$-strategy.

(ii) The composition of $\Lambda$-strategies is a $\Lambda$-strategy.

Proof. (i) The additional causal links introduced in the construction of the copycat strategy are between complementary events in $G^{\perp}$ and $G$, at the same level in $\Lambda$, and so respect $\leq$.

(ii) Let $\left(G, l_{G}\right),\left(H, l_{H}\right)$ and $\left(K, l_{K}\right)$ be $\Lambda$-games. Let $\sigma: G \gg H$ and $\tau: H \gg K$ be $\Lambda$-strategies. We show their composition $\tau \odot \sigma$ is a $\Lambda$-strategy.

It suffices to show $p \rightarrow p^{\prime}$ in $T \odot S$ implies $l_{G^{\perp} \| K} \tau \odot \sigma(p) \leq l_{G^{\perp} \| K} \tau \odot \sigma\left(p^{\prime}\right)$. Suppose $p \rightarrow p^{\prime}$ in $T \odot S$ with $\max (p)=e$ and $\max \left(p^{\prime}\right)=e^{\prime}$. Take $x \in \mathcal{C}(T \odot S)$ containing $p^{\prime}$ so $p$ too. Then, referring to Proposition 4 ,

$$
e \rightarrow \cup x e_{1} \rightarrow \cup x \cdots \rightarrow \cup x e_{n-1} \rightarrow \cup x e^{\prime}
$$

where $e, e^{\prime} \in V_{0}$ and $e_{i} \notin V_{0}$ for $1 \leq i \leq n-1$. ( $V_{0}$ consists of 'visible' events of the stable family, those of the form $(s, *)$ with $\sigma_{1}(s)$ defined, or $(*, t)$, with $\tau_{2}(t)$ defined.) The events $e_{i}$ have the form $\left(s_{i}, t_{i}\right)$ where $\sigma_{2}\left(s_{i}\right)=\tau_{1}\left(t_{i}\right)$, for $1 \leq i \leq n-1$.

Any individual link in the chain above has one of the forms:

$$
\begin{aligned}
& (s, t) \rightarrow \cup x\left(s^{\prime}, t^{\prime}\right),(s, *) \rightarrow \cup x\left(s^{\prime}, t^{\prime}\right), \\
& (*, t) \rightarrow \cup x\left(s^{\prime}, t^{\prime}\right),(s, t) \rightarrow \cup x\left(s^{\prime}, *\right), \quad \text { or } \quad(s, t) \rightarrow \cup x\left(*, t^{\prime}\right) .
\end{aligned}
$$

By Lemma 6, for any link either $s \rightarrow_{S} s^{\prime}$ or $t \rightarrow_{T} t^{\prime}$. As $\sigma$ and $\tau$ are $\Lambda$-strategies, this entails

$$
l_{G^{\perp} \| H} \sigma(s) \leq l_{G^{\perp} \| H} \sigma\left(s^{\prime}\right) \text { or } l_{H^{\perp} \| K} \tau(t) \leq l_{H^{\perp} \| K} \tau\left(t^{\prime}\right)
$$

for any link. Consequently $\leq$ is respected across the chain and $l_{G^{\perp} \| K} \tau \odot \sigma(p) \leq$ $l_{G^{\perp} \| K} \tau \odot \sigma\left(p^{\prime}\right)$, as required.

W.r.t. a particular choice of access levels $(\Lambda, \leq)$ we obtain a bicategory WLGames $_{\Lambda}$. Its objects are $\Lambda$-games $(G, l)$ where $G$ satisfies (Cwins) with arrows the $\Lambda$-strategies and 2-cells maps of spans. It restricts to a sub-bicategory of deterministic $\Lambda$-strategies, which as before is equivalent to an order-enriched category.

We can shift between different access levels. Let $r:(\Lambda, \leq) \rightarrow\left(\Lambda^{\prime}, \leq^{\prime}\right)$ be a monotonic function between preorders of levels. By composition with $r$ a $\Lambda$ game $(G, l)$ becomes a $\Lambda^{\prime}$-game $(G, r \circ l)$, giving rise to a (pseudo) functor from WGames $_{\Lambda}$ to WGames $_{\Lambda^{\prime}}$. Provided $r$ is injective, the functor has a right adjoint from WGames ${ }_{\Lambda^{\prime}}$ to WGames $_{\Lambda}$. 


\section{Acknowledgments}

Congratulations and thanks to Dexter Kozen for his dexterity across the areas of algorithmics and semantics. I'm grateful to the students at Aarhus who attended my course in the summer 2011, in particular to Nikoline Valsgaard Vinkel who bravely tackled the project on games with neutral positions. Thanks to my coworkers on the project ECSYM, Pierre Clairambault and Julian Gutierrez who in particular assisted in the Aarhus course. Thanks too to the anonymous referees for their helpful comments. The support of Advanced Grant ECSYM of the European Research Council is acknowledged with gratitude.

\section{References}

1. Winskel, G.: Event structures, stable families and games. Lecture notes, Comp Science Dept, Aarhus University, Available from http://daimi.au.dk/ gwinskel (2011)

2. Conway, J.: On Numbers and Games. Wellesley, MA: A K Peters (2000)

3. Winskel, G.: Event structure semantics for CCS and related languages. In: ICALP'82. Volume 140 of LNCS., Springer (1982)

4. Melliès, P.A.: Asynchronous games 2: The true concurrency of innocence. Theor. Comput. Sci. 358(2-3): 200-228 (2006)

5. Rideau, S., Winskel, G.: Concurrent strategies. In: LICS 2011, IEEE Computer Society (2011)

6. Clairambault, P., Gutierrez, J., Winskel, G.: The winning ways of concurrent games. Submitted (2012)

7. Winskel, G.: Events, causality and symmetry. Comput. J. 54(1) (2011) 42-57

8. Abramsky, S., Melliès, P.A.: Concurrent games and full completeness. In: LICS '99, IEEE Computer Society (1999)

9. Melliès, P.A., Mimram, S.: Asynchronous games : innocence without alternation. In: CONCUR '07. Volume 4703 of LNCS., Springer (2007)

10. Abramsky, S.: Semantics of interaction. In Pitts, A., Dybjer, P., eds.: Semantics and Logics of Computation. Publications of the Newton Institute (1997)

11. Hyland, M.: Game semantics. In Pitts, A., Dybjer, P., eds.: Semantics and Logics of Computation. Publications of the Newton Institute (1997)

12. Abramsky, S., Jagadeesan, R.: Game semantics for access control. Electronic Notes in Theoretical Computer Science 249 (2009) 135-156

13. Joyal, A.: Remarques sur la théorie des jeux à deux personnes. Gazette des sciences mathématiques du Québec, 1(4) (1997)

14. Hintikka, J., Sandu, G.: A revolution in logic? Nordic J. of Phil. Logic 1 (1996)

15. Clairambault, P., Gutierrez, J., Winskel, G.: Imperfect information in logic and concurrent games. Submitted (2012) 